\title{
Vaccine Hesitancy Counseling-an Educational Intervention to Teach a Critical Skill to Preclinical Medical Students
}

\author{
Arati Kelekar $^{1}$ (D) $\cdot$ Ida Rubino $^{1} \cdot$ Maurice Kavanagh $^{1} \cdot$ Robin Lewis-Bedz $^{2} \cdot$ Gabrielle LeClerc $^{1} \cdot$ Leon Pedell $^{1}$. \\ Nelia Afonso ${ }^{1}$
}

Accepted: 8 December 2021 / Published online: 21 January 2022

(c) The Author(s) under exclusive licence to International Association of Medical Science Educators 2021

\begin{abstract}
Introduction There has been a recent rise in public perception that vaccines are unsafe, fostering vaccine hesitancy (VH). Few interventions have focused on teaching medical students' communication skills for counseling vaccine-hesitant patients. Methods Our educational intervention, designed for medical students, involved a self-study module followed by an interactive session on VH. Students practiced counseling vaccine-hesitant standardized patients (SPs). Faculty and SPs assessed student counseling skills. Students completed pre- and post-intervention surveys to assess attitudes and preparedness to counsel VH patients.

Results Students showed a better ability to talk to parents about Human Papillomavirus (HPV) vaccine concerns and Measles Mumps Rubella (MMR)/autism-related issues than to address patients' concerns related to the Varicella Zoster Virus (VZV) vaccine.

Students' surveys pre- and post-intervention revealed significant improvement in their vaccination knowledge and comfort with counseling vaccine-hesitant patients. Student counseling skills as part of an Objective Structured Clinical Exam (OSCE) showed $73 \%$ of students asked about immunization and elicited SP concerns, but only $36 \%$ counseled appropriately.

Conclusions In the face of emerging VH, physicians play a critical role in advising and influencing vaccination decisions. Therefore, it is a core responsibility of medical educators to train medical students on recommending vaccinations and responding effectively to vaccine-hesitant parents and patients. Our multifaceted interactive session provided preclinical students with knowledge and skills to improve communication skills with $\mathrm{VH}$ patients and parents and the need for ongoing practice of these VH counseling skills.
\end{abstract}

Keywords Vaccine hesitancy $\cdot$ Education intervention $\cdot$ Simulation $\cdot$ Medical students

\section{Introduction}

Vaccines are safe, effective, and one of the most successful forms of prevention [1]. The proliferation of vaccine scares and controversies in the media has resulted in a significant public perception that vaccines are unsafe, fostering vaccine hesitancy $(\mathrm{VH})$ resulting in populations with declining immunity and outbreaks of vaccinepreventable diseases. According to the World Health

Arati Kelekar

Arati.Kelekar@beaumont.org

1 Oakland University William Beaumont School of Medicine, Rochester, Michigan 48309, USA

2799 W Grand Blvd, Detroit, Michigan 48202, USA
Organization, VH is defined as a delay in acceptance or refusal of vaccines despite availability of vaccination services [2]. Vaccine attitudes range from full acceptance to complete refusal. Healthcare providers remain a credible source of vaccine information. Research has shown that patients who receive a strong recommendation from a healthcare provider are $4-5$ times more likely to be vaccinated [3]. The National Vaccine Advisory Committee: "Standards for Adult Immunization Practice" states the following: "One of the most important predictors of vaccination receipt among adults is a health-care provider's recommendation and offer of a vaccine during the same visit." [4].

In the pediatric context, parents were twice as likely to respond to healthcare providers who affirmed the safety of vaccines for their children [5]. It is therefore important 
to provide medical trainees with the appropriate communication tools for vaccination discussions. During pediatric rotations, few medical students witnessed the provider initiation of vaccine discussions with strong vaccine recommendations [6] Real et al. demonstrated the success of a residency curriculum focused on communication strategies with patients hesitant about the influenza vaccine, finding a decreased rate of vaccination refusal in the post curricular period [7].

There are only a few published reports of educational interventions that focus on specifically teaching medical students' communication skills for counseling vaccine-hesitant patients and parents. These range from an art-based video format helping preclinical students reflect on the health beliefs raised by vaccine-hesitant parents [8] to flipped classroom and case-based format for third-year students [9]. Schnaith et al. showed an educational intervention followed by role-play resulted in an increase in student awareness about the HPV vaccine as well as improved student comfort level discussing this with vaccine-hesitant parents [10]. A survey of French medical students concluded that practical training methods like case-based learning and clinical placements resulted in better self-perceived preparedness to respond to questions about vaccines [11].

At our medical school, several curricular initiatives have been developed to educate medical students about vaccines. These curricular interventions and training strategies were developed based on our research on vaccine attitudes among medical students $[12,13]$. Our multifaceted influenza vaccine educational intervention in 2011 offered first-year medical students experience with vaccine counseling and administration and positively influenced student attitudes towards influenza [12]. In 2013, we surveyed our students on their knowledge and attitudes towards HPV [13]. Notably, only $40 \%$ felt they possessed sufficient information to adequately counsel patients and similarly only $40 \%$ felt comfortable counseling patients. Increased positive attitudes and better knowledge scores were noted in vaccinated students compared to non-vaccinated students. This impelled us to design this educational intervention to teach counseling to VH patients.

We designed a curriculum to offer a real-world simulation of VH through a standardized patient (SP) encounter followed by SP and faculty feedback on the practiced skills.

The objectives of our study were to:

1. Develop a curricular intervention to improve student knowledge and skills when communicating with vaccine-hesitant patients.
2. Evaluate personal attitudes and preparedness to discuss VH pre- and post-intervention.

3. Evaluate retention of skills during objective structured clinical examination (OSCE) conducted a year after the educational intervention.

\section{Methods}

The University Institutional Review Board (IRB numbers 1473508-2, 1,537,559-1) approved the study protocol.

\section{Setting and Participants}

Second-year medical (M2) students enrolled in an allopathic medical school $(n=126)$ during the academic year 2019-2020.

\section{Design of Educational Intervention}

This educational intervention was based on a synthesis of instructional design theories as proposed by Merrill [14] who postulated that student learning will be promoted when (1) instruction is problem- or task-centered, (2) student learning is activated by connecting what they already know or can do with what is to be newly learned, (3) students are exposed to demonstrations of what they are to learn, (4) they have opportunities to try out what they have learned with instructor coaching and feedback, and (5) they integrate what they have learned into their personal lives [14] (Fig. 1). We selected VH scenarios that represented discussions commonly encountered with patients/parents across different age groups: Measles Mumps Rubella (MMR) vaccine during infancy, Human Papillomavirus (HPV) vaccine during pre-adolescence, and Varicella Zoster (VZV) vaccine in adults.

\section{Pre-reading}

A self-study module included information regarding $\mathrm{VH}$ myths about MMR and autism, the retracted Wakefield study [15], and resources from the Centers for Disease Control and Prevention, including information sheets on HPV, MMR, and VZV vaccines. 
Fig. 1 Educational intervention

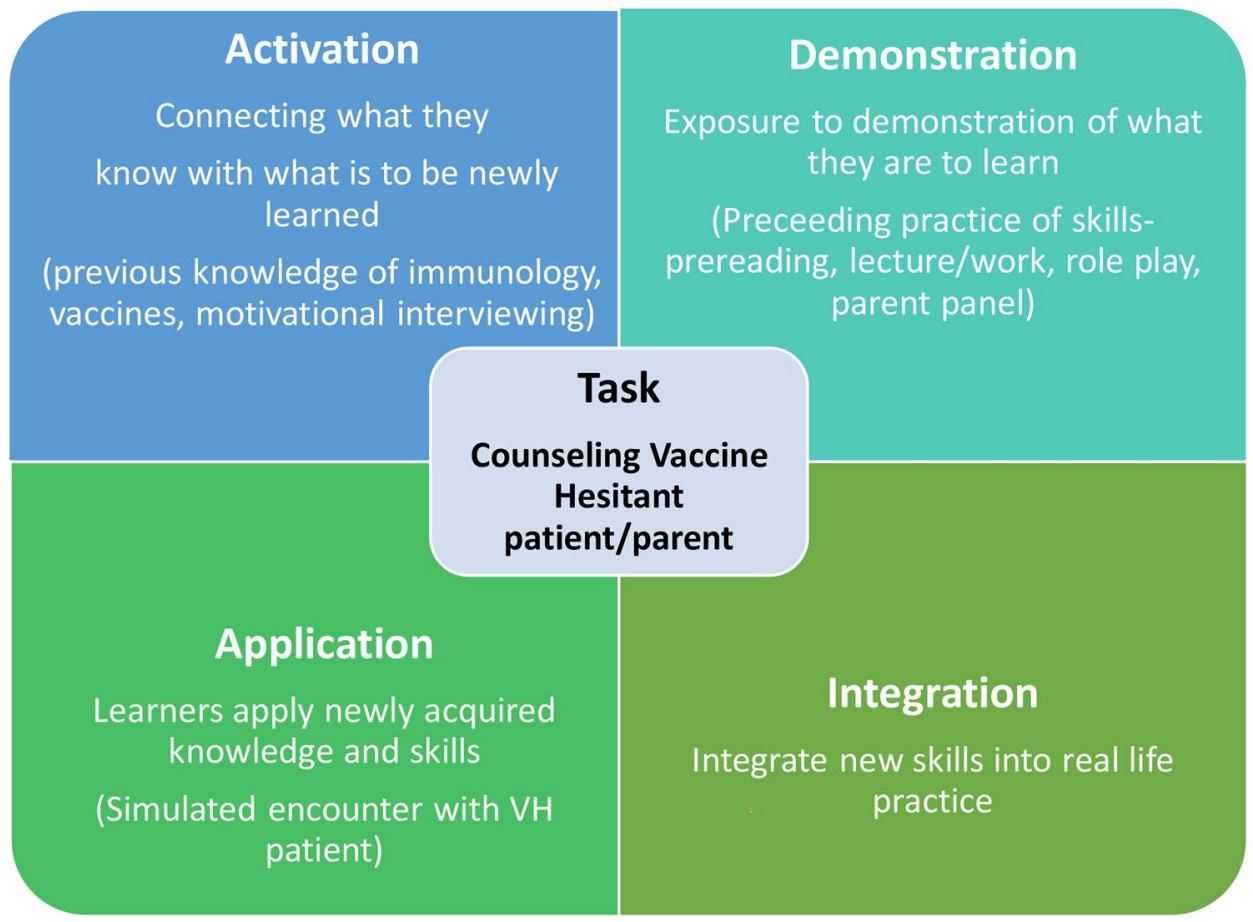

\section{Vaccine Hesitancy Session}

1. Patient panel with parents who had lost children due to vaccine-preventable illnesses and subsequently dedicated themselves to spreading awareness about vaccines (The Emily Stillman Foundation and Alana's Foundation).

2. Didactic lecture and interactive workshop delivered by faculty discussed $\mathrm{VH}$ misconceptions, use of motivational interviewing skills with vaccine-hesitant patients and parents, and guidelines for documentation of vaccine refusal and waiver. Information pertaining to the HPV, MMR, and VZV vaccine was reviewed.

\section{Simulated VH Encounter with Standardized Patients (SPs)}

Following the VH lecture, students in small groups $(n \sim 6)$ practiced counseling skills with SPs. Three vaccine scenarios were developed by the faculty associated with the clinical skills course. A pair of students in each group was assigned to interview an SP who received standardized training to roleplay each of the scenarios. The SPs were instructed to bring up specific myths associated with each vaccine, and students were expected to address patient/parental concerns, providing education and counseling while maintaining a non-judgmental attitude. Following each encounter, SPs provided feedback from a patient's perspective of the students' interaction with them as well as specific communication skills. The faculty received facilitator guides that included instructions on scoring the clinical information discussed by the student. They also provided feedback on overall communication skills. Both faculty and SPs completed a grading rubric.

\section{VH Scenarios}

1. Parent with concerns about HPV vaccine.

2. Young mother with MMR concerns who has not approved any vaccinations for her child.

3. Older adult questioning the need for a "shingles" (VZV) vaccine.

\section{Pre/Post-intervention Survey}

Prior to the intervention, students were asked to complete a voluntary online 8-item 5-point Likert attitude survey designed to measure baseline confidence in their knowledge of, and ability to address, VH. Previous research on VH in healthcare workers assisted in formulating the content of the questionnaire $[11,16]$. An identical post-intervention survey was administered 2 weeks later.

\section{Retention of VH Counseling Skills 1 Year After Intervention}

One of the stations of the M3 mid-year OSCE was a comprehensive history on a patient presenting with back pain who also had concerns about the influenza vaccine. Students were evaluated by SPs who were asked to specifically note whether students had asked them about immunization, elicited their concerns about the influenza vaccine, and counseled them appropriately. 
Table 1 Vaccine hesitancy encounter-faculty rating of clinical information

\begin{tabular}{|c|c|c|c|c|}
\hline Vaccine & $\mathrm{VZV}^{*}(N=42)$ & $\operatorname{HPV}^{* *}(N=41)$ & $\operatorname{MMR} * * *(N=43)$ & Kruskal-Wallis \\
\hline Knowledge items & $\begin{array}{l}\text { - Causes chicken pox and shingles } \\
\text { - Connection between chickenpox } \\
\text { and shingles/post herpetic neuralgia } \\
\text { - Effectiveness of vaccines } \\
\text { - Recommendations }\end{array}$ & $\begin{array}{l}\text { - Link between HPV and } \\
\text { cancer } \\
\text { - HPV cause of anogenital } \\
\text { or oropharyngeal cancer } \\
\text { - HPV vaccine for cancer } \\
\text { prevention } \\
\text { - Recommendations }\end{array}$ & $\begin{array}{l}\text { - MMR no links to autism } \\
\text { (Wakefield study redacted) } \\
\text { - Safety of thimerosal } \\
\text { - Safety of multiple child- } \\
\text { hood vaccine administra- } \\
\text { tion } \\
\text { - Safety of MMR }\end{array}$ & \\
\hline Total (out of 8) & $5.55 / 8.00$ & $6.98 / 8.00$ & $7.06 / 8.00$ & $H(2)=23.73, p<0.01$ \\
\hline Scale & $\begin{array}{l}\text {-Well discussed at patient level (2) } \\
\text {-Partially communicated (1) } \\
\text { - Not addressed (0) }\end{array}$ & & & \\
\hline
\end{tabular}

SP scenarios:

*Older adult questioning the need for a "shingles" (VZV) vaccine

** Parent with concerns about HPV vaccine

**** Mother with MMR concerns who has not approved any vaccinations for her child

\section{Data and Analysis}

All statistical analysis were performed using SPSS version 25.

\section{SP and Faculty Ratings of Student Counseling Behaviors and Knowledge}

For each of the three scenarios, SPs and faculty concurrently rated students. SPs rated students on communication skills-14 questions were collapsed into agenda building (maximum 3 points), validation (maximum 5 points), knowledge (maximum 4 points), and conclusion (maximum 2 points). Faculty scored students on their knowledge and accuracy of facts conveyed to the SPs (maximum 8 points). Between scenario scores were compared using the Kruskal-Wallis $H$ test for differences between medians; nominal alpha was set at 0.01 . SP and faculty ratings were recorded and analyzed independent of and separate from one another.

\section{Pre- and Post-intervention Surveys}

Comparison was performed using paired samples $T$-test.

\section{Results}

Of the 126 students attending the VH session, 42 completed the VZV scenario, 41 completed the HPV scenario, and 43 completed the MMR scenario.

\section{Faculty Evaluation of VH Encounter}

Mean faculty scores ranged from 5.55/8 (69.35\%) for the VZV scenario, 6.98/8 (87.2\%) for the HPV vaccine, to 7.06/8 (88.23\%) for the MMR scenario. The Kruskal-Wallis $H$ $(2)=23.73, p<0.01$ indicated the overall difference between groups was significant at the alpha 0.01 level, with students

Table 2 Vaccine hesitancy encounter-SP rating of communication behaviors

\begin{tabular}{lllll}
\hline Vaccine & $\mathrm{VZV}^{*}(N=42)$ & $\mathrm{HPV} * *(N=41)$ & $\mathrm{MMR} * * *(N=43)$ & $\mathrm{Kruskal}-\mathrm{Wallis}$ \\
\hline $\begin{array}{l}\text { Established agenda } \\
\text { (maximum 3 points) }\end{array}$ & 2.1 & $2.8394 .31 \%$ & $2.8695 .35 \%$ & $H(2)=46.94, \mathrm{p}<.01$ \\
$\begin{array}{l}\text { Affirmed/validated my concerns } \\
\text { (maximum 5 points) }\end{array}$ & $69.84 \%$ & $2.2745 .37 \%$ & $2.3747 .44 \%$ & $H(2)=.10, p=.95$ \\
$\begin{array}{l}\text { Provided medical information about vaccine } \\
\text { (maximum 4 points) }\end{array}$ & $1.8847 .02 \%$ & $3.7192 .68 \%$ & $3.0977 .33 \%$ & $H(2)=76.82, p<0.01$ \\
\begin{tabular}{l} 
Conclusion of the interview (maximum 2 points) \\
\hline
\end{tabular} & $1.4572 .62 \%$ & $1.6180 .49 \%$ & $1.7788 .37 \%$ & $H(2)=3.80, p=.15$ \\
\hline
\end{tabular}

SP scenarios:

*Older adult questioning the need for a "shingles" (VZV) vaccine

** Parent with concerns about HPV vaccine

**** Mother with MMR concerns who has not approved any vaccinations for her child 
assigned to the VZV case scoring significantly lower than those assigned to either HPV or MMR cases (Table 1).

\section{SP Rating of Communication Behaviors During VH Encounter}

There were no significant differences in the students' abilities to validate the SP concerns at the conclusion of the 3 scenarios (Table 2). However, there were significant differences in SP ratings of agenda setting and medical information provided in the VZV scenario as compared to the HPV and MMR scenarios.

\section{Student Pre- and Post-intervention Survey}

Forty-three of the 126 students took the pre-intervention survey and 44 of the 126 took the post-intervention survey. Of these, 20 pairs (16\%) of matched surveys were analyzed and results are shown in Table 3. Post intervention there were significant increases in student perception of their preparation to recommend vaccines, having adequate knowledge about vaccine hesitancy, ability to communicate with vaccine-hesitant patients, using evidence-based information to counteract messages that misinform the public about vaccines and comfort with documentation of vaccination refusal.

There were no significant increases in the student perception of trust in Centers for Disease Control and Prevention (CDC) recommendations or their perception that $\mathrm{CDC}$ vaccine recommendations should be mandatory.

\section{Retention of VH Counseling Skills 1 Year After Intervention}

One hundred and twenty-four students obtained a comprehensive history as part of the OSCE. Ninety students (72.6\%) asked about immunizations. Forty-five students (36.3\%) counseled on $\mathrm{VH}$ concerns, while 79 (63.7\%) did not provide any counseling.

Table 3 Pre-post survey responses

\begin{tabular}{|c|c|c|c|c|c|c|c|c|c|}
\hline $\begin{array}{l}\text { Survey item (average score out of } 5,1=\text { strongly } \\
\text { disagree, } 5=\text { strongly agree) }\end{array}$ & Pre & Post & Change & Std Dev & Lower 95\% CI & Upper 95\% CI & $t$ & DF & $\overline{\text { Sig ( } 2 \text { tailed })}$ \\
\hline Vaccines are safe & 4.85 & 4.90 & .05 & 0.22 & -0.15 & 0.05 & -1.00 & 19 & 0.33 \\
\hline $\begin{array}{l}\text { I trust the Centers for Disease Control and Pre- } \\
\text { vention }(\mathrm{CDC}) \text { vaccine recommendations }\end{array}$ & 4.80 & 4.95 & .15 & 0.37 & -0.32 & 0.02 & -1.83 & 19 & 0.083 \\
\hline $\begin{array}{l}\text { I believe all CDC recommendations should be } \\
\text { mandatory }\end{array}$ & 4.15 & 4.40 & .25 & 0.72 & -0.59 & 0.09 & -1.56 & 19 & 0.135 \\
\hline $\begin{array}{l}\text { I feel adequately prepared to recommend vac- } \\
\text { cines to patients and parents }\end{array}$ & 3.75 & 4.65 & .90 & 1.07 & -1.40 & -0.40 & -3.76 & 19 & $0.001 *$ \\
\hline $\begin{array}{l}\text { I feel I have adequate knowledge about vaccine } \\
\text { hesitancy }\end{array}$ & 4.05 & 4.75 & .70 & 0.80 & -1.08 & -0.32 & -3.91 & 19 & $0.001^{*}$ \\
\hline $\begin{array}{l}\text { I feel comfortable communicating with vaccine } \\
\text { hesitant patients/ parents }\end{array}$ & 3.60 & 4.55 & .95 & 0.69 & -1.27 & -0.63 & -6.19 & 19 & $<.001 *$ \\
\hline $\begin{array}{l}\text { I feel comfortable using evidence-based informa- } \\
\text { tion to counteract messages that can misinform } \\
\text { the public about vaccines }\end{array}$ & 4.10 & 4.80 & .70 & 0.57 & -0.97 & -0.43 & -5.48 & 19 & $<.001 *$ \\
\hline $\begin{array}{l}\text { I feel comfortable documenting vaccination } \\
\text { refusals in the medical record }\end{array}$ & 3.40 & 4.20 & .80 & 1.15 & -1.34 & -0.26 & -3.11 & 19 & $0.006^{*}$ \\
\hline
\end{tabular}

Test is a paired samples $t$-test. Software used is SPSS

Key, left to right

Pre: average pre-intervention score (out of 5)

Post: average post-intervention score (out of 5)

Change: difference between pre- and post-intervention

Std Dev: standard deviation

Lower 95\% CI: lower limit 95\% confidence interval

Upper 95\% CI: upper limit 95\% confidence interval

$T$ : paired samples $t$ score

DF: degrees of freedom $(N=20)$

Sig (2 tailed): two tailed significance

Items marked with * differ significantly at alpha .01 level 


\section{Discussion}

In the face of emerging $\mathrm{VH}$, physicians play a critical role in advising and influencing vaccination decisions. Therefore, it is a core responsibility of medical educators to train medical students on recommending vaccinations and responding effectively to vaccine-hesitant parents and patients. Previous studies have shown that medical students and residents receive insufficient training and are not adequately prepared to communicate with their patients about vaccines, vaccine myths, and strategies to respond to vaccine-hesitant patients or parents $[11,17]$.

To the authors' knowledge, this is the first study that has evaluated medical students' communication skills related to VH after an educational intervention using SPs. Our multifaceted educational intervention was unique, since it gave students an opportunity to practice their communication skills following instruction about $\mathrm{VH}$ in a classroom setting. The parents on the vaccine panel who recounted their personal experiences with devastating consequences of vaccinepreventable diseases were also an important aspect of this educational intervention allowing students to gain insight on the patient's perspective. The subsequent VH encounter enabled faculty and SPs to assess several undergraduate medical education (UGME) core competencies and also facilitate real-time coaching and feedback. Communication strategies specifically recommended for conversations involving $\mathrm{VH}$, such as motivational interviewing techniques and avoidance of adversarial conversations, were emphasized [18].

In our study, SPs rated the students' ability to communicate medical information about HPV and MMR vaccines higher compared to the VZV vaccine. Faculty assessment of student knowledge during the encounter correlated with the $\mathrm{SP}$ assessment. This illustrates the fact that, in addition to communication skills, medical knowledge of the vaccine is a critical component when counseling VH patients. Cvjetkovic et al. found significant gaps in knowledge among medical students with respect to complications of diseases protected by vaccines; this could explain the relative misconceptions observed during counseling involving the VZV vaccine [19].

It is not very clear to the authors why student knowledge about HPV and MMR vaccines was better than that about the VZV vaccine since the educational intervention provided comparable information on all 3 vaccines. As shown in previous studies, this could be attributed to the association between vaccine-related attitudes affected by personal experience among our students who had received MMR and likely received HPV vaccine, but had no personal experience with the VZV vaccine [13, 20]. Hurley et al. have also reported inconsistencies in the understanding of the precise indications for VZV vaccine among primary care physicians [21]. We need to improve the clarity of educational materials to assist students in understanding the pathophysiological mechanisms involved in the protection afforded by the vaccine. This educational aspect will be especially important in the context of SARS-Cov-2 infection as multiple vaccines with varying immunological mechanisms of action become available.

The pre and post surveys suggest this educational intervention significantly improved students' self-reported perception of their ability to recommend vaccines and communicate with vaccine-hesitant patients, as well as their comfort with documentation of vaccination refusal.

A year later, student counseling skills regarding $\mathrm{VH}$ were assessed. Although $73 \%$ of students asked about the influenza vaccine and elicited SP concerns, only a $36 \%$ counseled appropriately. Possible reasons for this poor performance could be attributed to the time constraints during the OSCE and the fact that $\mathrm{VH}$ was not the primary focus of the encounter during the OSCE.

This study has several limitations. There was only a single practice session with SPs, and students did not get an opportunity for further practice to refine these $\mathrm{VH}$ counseling skills. There was a low response rate to the survey $(16 \%$ of matched pairs). These responses may not reflect the views of the entire group. Although these limitations exist, important conclusions can be gained from this educational intervention. Primarily, this intervention demonstrated that it is possible to teach preclinical medical students VH communication skills in a simulated setting, providing them with a strong foundation of $\mathrm{VH}$ counseling skills before they begin clerkships.

Our intervention provided faculty an opportunity to observe students perform activities related to Association of American Medical College's Core Entrustable Professional Activities 4 and 11 (AAMC EPA's) as well as several competencies related to Patient Care (PC), Knowledge for Practice (KP), and Interpersonal and Communication Skills (ICS). Continued education and interventions are needed as they progress, so that all medical students graduate with the ability to provide strong vaccine recommendations. We plan on assessing these skills longitudinally as this student cohort progresses through clerkships, and anticipate improvement after clinical exposure. The recent pandemic has underscored the global threat of $\mathrm{VH}$. We have continued to refine this program and added additional scenarios with COVID-19 VH and provided additional opportunities for students to practice these counseling skills by participating in community vaccine education programs.

\section{Conclusion}

This multifaceted educational intervention demonstrated the importance of both knowledge and communication skills needed during interactions with VH patients. We need to equip our future healthcare providers with appropriate skills to make informed vaccine recommendations and to respond 
effectively to vaccine-hesitant patients. We hope this unique educational intervention, targeting preclinical medical students, will enable them to address these challenging conversations with patients and parents.

Acknowledgements The authors wish to thank Emily Stillman Foundation (https://www.foreveremily.org/L and Alana's Foundation (https:// www.alanasfoundation.org/) for their participation in the educational session as part of parent panel.

Author Contribution Arati Kelekar-conceptualization, methodology, writing original draft-lead, review and editing. Ida Rubinoconceptualization, methodology, writing - review and editing. Gabrielle LeClerc—data curation, project administration, writing - review and editing. Robin Lewis-Bedz-methodology, data curation, project administration. Maurice Kavanagh — conceptualization, methodology, data analysis-lead. Leon Pedell—conceptualization, methodology, writing-review and editing. Nelia Afonso-conceptualizationlead, methodology, writing-review and editing.

Availability of Data and Material Not applicable.

Code Availability Not applicable.

\section{Declarations}

Ethics Approval The University Institutional Review Board (IRB numbers 1473508-2, 1537559-1) approved the study protocol.

Consent to Participate Informed consent was obtained from all students prior to participation in survey.

Consent for Publication Not applicable (no identifiable information included in the publication).

Conflict of Interest The authors declare no competing interests.

\section{References}

1. National Infant Immunization Week Overview: Milestones Reached. Centers for Disease Control and Prevention website. https://www.cdc.gov/vaccines/events/niiw/overview.html Updated April 2, 2020. Accessed 11 July, 2021

2. World Health Organization. Report of the Sage Working Group on Vaccine Hesitancy. 2014. https://www.who.int/immunization/sage/ meetings/2014/october/1_Report_WORKING_GROUP_vaccine_ hesitancy_final.pdf Updated October 1, 2014. Accessed 11 July, 2021

3. Lau M, Hua L, Flores G. Factors associated with human papillomavirus vaccine-series initiation and healthcare provider recommendation in US adolescent females: 2007 National Survey of Children's Health. Vaccine. 2012;30(20):3112-8. https://doi.org/ 10.1016/j.vaccine.2012.02.034.

4. Committee NVA. Recommendation from the National Vaccine Advisory Committee: standards for adult immunization practice. Public Health Rep. 2014;129(2):115-23. https://doi.org/10.1177/ 003335491412900203.

5. Smith PJ, Kennedy AM, Wooten K, Gust DA, Pickering LK. Association between health care providers' influence on parents who have concerns about vaccine safety and vaccination coverage. Pediatrics. 2006;118(5):e1287-92. https://doi.org/10.1542/peds.2006-0923.
6. Caruso Brown AE, Suryadevara M, Welch TR, Botash AS. "Being persistent without being pushy": student reflections on vaccine hesitancy. Narrat Inq Bioethics. 2017;7(1):59-70. https://doi.org/ 10.1353/nib.2017.0018.

7. Real FJ, DeBlasio D, Beck AF, et al. A virtual reality curriculum for pediatric residents decreases rates of influenza vaccine refusal. Acad Pediatr. 2017;17(4):431-5. https://doi.org/10.1016/j.acap.2017.01.010.

8. Koski K, Lehto JT, Hakkarainen K. Simulated encounters with vaccine-hesitant parents: arts based video scenario and a writing exercise. J Med Educ Curric Dev. 2018;5:2382120518790257. https://doi.org/10.1177/2382120518790257.

9. Coleman A, Lehman D. A flipped classroom and case-based curriculum to prepare medical students for vaccine-related conversations with parents. MedEdPORTAL. 2017;13:10582. https://doi. org/10.15766/mep_2374-8265.10582

10. Schnaith AM, Evans EM, Vogt C, et al. An innovative medical school curriculum to address human papillomavirus vaccine hesitancy. Vaccine. 2017;36(26):3830-5. https://doi.org/10.1016/j. vaccine.2018.05.014.

11. Kernéis S, Jacquet C, Bannay A, May T, Launay O, Verger P, Pulcini C. Vaccine education of medical students: a nationwide cross-sectional survey. Am J Prev Med. 2017;53(3):e97-104. https://doi.org/10.1016/j. amepre.2017.01.014.

12. Afonso N, Kavanagh M, Swanberg S. Improvement in attitudes towards influenza vaccination in medical students following an integrated curricular intervention. Vaccine. 2014;32(4):502-6. https://doi.org/10.1016/j.vaccine.2013.11.043.

13. Afonso NM, Kavanagh MJ, Swanberg SM, Schulte JM, Wunderlich $\mathrm{T}$, Lucia VC. Will they lead by example? Assessment of vaccination rates and attitudes to human papilloma virus in millennial medical students. BMC Public Health. 2017;17(1):35. https://doi.org/10. 1186/s12889-016-3969-x.

14. Merrill MD. First principles of instruction. Education Technology Research \& Development. 2002;50(3):43-59. https://doi.org/10. 1007/BF02505024.

15. Wakefield AJ, Murch SH, Anthony A, et al. RETRACTED: Ileallymphoid-nodular hyperplasia, non-specific colitis, and pervasive developmental disorder in children. Lancet. 1998;351(9103):63741. https://doi.org/10.1016/s0140-6736(97)11096-0.

16. Larson HJ, Jarrett C, Schulz WS, et al. Measuring vaccine hesitancy: the development of a survey tool. Vaccine. 2015;33(34):4165-75. https://doi.org/10.1016/j.vaccine.2015.04.037.

17. Leask J, Kinnersley P, Jackson C, Cheater F, Bedford H, Rowles G. Communicating with parents about vaccination: a framework for health professionals. BMC Pediatr. 2012;12:154. https://doi. org/10.1186/1471-2431-12-154.

18. Williams SE, Swan R. Formal training in vaccine safety to address parental concerns not routinely conducted in U.S. pediatric residency programs. Vaccine. 2014;32(26):3175-8. https://doi.org/ 10.1016/j.vaccine.2014.04.001

19. Cvjetkovic SJ, Jeremic VL, Tiosavljevic DV. Knowledge and attitudes toward vaccination: a survey of Serbian students. J Infect Public Health. 2017;10(5):649-56. https://doi.org/10.1016/j.jiph.2017.05.008.

20. Finney Rutten LJ, St Sauver JL, et al. Clinician knowledge, clinician barriers, and perceived parental barriers regarding human papillomavirus vaccination: association with initiation and completion rates. Vaccine. 2017;35(1):164-9. https://doi.org/10. 1016/j.vaccine.2016.11.012.

21. Hurley LP, Allison MA, Dooling KL, et al. Primary care physicians' experience with zoster vaccine live (ZVL) and awareness and attitudes regarding the new recombinant zoster vaccine (RZV). Vaccine. 2018;36(48):7408-14. https://doi.org/10.1016/j.vaccine.2018.09.018.

Publisher's Note Springer Nature remains neutral with regard to jurisdictional claims in published maps and institutional affiliations. 